\title{
Sudden Unexpected Infant Death: Review and Analysis of Adherence to Recommendations
}

Enrique Konstat-Korzenny ${ }^{1}$, Ariel Cohen-Welch ${ }^{2}$, Rodrigo Fonseca-Portilla ${ }^{2}$, Dan Morgenstern-Kaplan ${ }^{2}$

1. Centro De Investigación En Ciencias De La Salud Anáhuac (CICSA), Facultad De Ciencias De La Salud, Universidad Anahuac Mexico, Mexico City, MEX 2. Centro De Investigación En Ciencias De La Salud Anáhuac (CICSA), Facultad De Ciencias De La Salud, Universidad Anáhuac Mexico, Mexico City, MEX

Corresponding author: Dan Morgenstern-Kaplan, dmorgensternk@gmail.com

\section{Abstract}

\section{Introduction}

Sudden Unexpected Infant Death (SUID) is a term that englobes the sudden and unexpected death of an infant less than 12 months, which can be explained by organic or traumatic causes, or that can't be explained such as cases of Sudden Infant Death Syndrome (SIDS). Although many risk factors have been associated with this syndrome, one of the most widely associated and studied are incorrect sleeping techniques and practices. In 2016, the American Academy of Pediatrics (AAP) updated their safe-sleeping guidelines and sleeping environment recommendations and strategies to prevent SIDS.

\section{Methods}

We conducted a cross-sectional study to analyze the sleeping environments in infants that attended both the inpatient and outpatient services at a public pediatric hospital in Mexico City. A 6-item questionnaire was applied to the parent or guardian to assess the sleeping habits of infants in their homes.

\section{Results}

A total of 184 infants were included in the study, with a mean age of 5.87 months. Overall, the number of parents that follow safe sleeping practices was very low, with no parents following all the AAP recommendations, and over $10 \%$ not following any. Although there is uncertainty about the pathogenesis of this syndrome, the focus has shifted to prevention, especially regarding the modifiable risk factors.

\section{Conclusions}

It was demonstrated that our population did not know about the proper and safe sleeping techniques. In the nearby future, our goal is for health authorities in our country to implement a strategy to make the AAP recommendations part of government health campaigns.

Received 10/08/2019

Review began 10/14/2019

Review ended 10/31/2019

Published 11/05/2019

\section{() Copyright 2019}

Konstat-Korzenny et al. This is an open access article distributed under the terms of the Creative Commons Attribution License CC-BY 3.0., which permits unrestricted use, distribution, and reproduction in any medium, provided the original author and source are credited.
Categories: Pediatrics, Epidemiology/Public Health

Keywords: pediatrics, sleeping practices, sids, safety, suid, safe sleeping

\section{Introduction}

Sudden Unexpected Infant Death (SUID) is a term that englobes the sudden and unexpected death of an infant less than 12 months of age such that after a thorough investigation of the case, the cause of death was due to suffocation, entrapment, infection, ingestion, metabolic diseases, arrhythmias, trauma or Sudden Infant Death Syndrome (SIDS; term for deaths that remain unexplained even after thorough investigation of the case and scene, clinical history, and autopsy of the body) [1-2].

\section{Epidemiology}

According to the American Academy of Pediatrics (AAP), more than 3,500 infants die annually due to SUID and sleep-related deaths [3]. In 2010, it was reported that 1 out of 3 postnatal deaths, and 1 out of 7 infant deaths were attributed to SUID [4]. The Center for Disease Control and Prevention (CDC) reported 3,600 SUID cases in 2017, further dividing it into SIDS (38\%), accidental suffocation and strangulation in bed (26\%) and unknown causes (36\%) [5]. The incidence of SUID seems to be more prevalent in the African American, American Indian and Native Alaskan population [3]. Since the establishment of safe-sleeping practices campaigns across the world, the incidence of SUID has dramatically fallen. An approximate decrease of 50\% was reported in SUID since the mid 1980's [6].

\section{Pathogenesis}

There is no definite pathophysiological process that can be attributed to SUID. It is thought to be a combination of various factors occuring in conjunction that lead to the lethal event. Normally, there are 
previous conditions that can increase the infant's vulnerability, such as premature birth and birth defects triggering the cause of death, whether an infectious, respiratory or metabolic cause. These factors, along with an immature immunologic or nervous system, predispose the child to a higher risk of SUID [7].

\section{Risk factors for SUID}

Birth Weight and Age

Among the infant-related risk factors, low birth weight and preterm birth are the most remarkable. In one study, preterm infants had a higher risk of SUID than term infants [8]. Small for Gestational Age (SGA) infants also had a slightly increased risk of SUID (1.65 OR (95\% CI 1.47 to 1.85)), while Large for Gestational Age (LGA) infants had a reduced risk of SUID (0.73 (CI 0.60 to 0.89 ) mortality, although the mechanisms behind these phenomena are uncertain [9].

\section{Sleeping Position}

Sleeping position is another important risk factor and the main focus of many SUID prevention campaigns [6,10]. Non-supine or prone sleeping positions are associated with a higher risk of SUID [3,11]. One study that analyzed 11,717 infant death cases, where $27.4 \%$ classified as SUID, prone sleeping was reported in up to $42.4 \%$ of the deaths [12]. A recent study gathered polysomnographic information, documenting that infants during prone sleeping had a higher heart rate, decreased oxygen saturation and more time with oxygen saturation below $90 \%$, compared with infants who were sleeping in the supine position, offering possible explanations behind the physiopathology of inadequate sleeping positions and their relation to SUID [13].

Sleeping Environment

Loose bedding or objects in the immediate sleeping environment of the infant are also associated with SUID. One study reported that 14\% of SUID cases reported by the CDC from 2011-2014 were attributed to suffocation, and $69 \%$ of those deaths were attributed to loose bedding [14]. One systematic review gathered information about 10 SUID studies and concluded that almost $25 \%$ of cases were related to loose bedding [15].

Sharing beds with another person (bed sharing) and co-sleeping are common practices throughout the world, especially in underdeveloped countries. While some benefits have been reported, mainly related to improved breastfeeding and increased emotional bond between mother and infant [16-18], many experts and studies advise against it because of its association with SUID. A meta-analysis concluded that cosleeping increased the risk of SUID, even if parents did not smoke. [19] Various other studies and metaanalysis have demonstrated an increased risk with these practices [12,20]. Sharing a sleeping surface, other than a bed, such as a sofa, has also been studied and presents a high risk of SUID [21].

Maternal or Environmental Risk Factors

Smoking during pregnancy or after birth is another important risk factor for SUID [20]. A twofold increase in SUID risk was reported in infants born to mothers who smoked during pregnancy, as well as a linear increase in the risk of SUID with each additional cigarette smoked during pregnancy [22]. An increased risk was also reported in infants whose parents were passive smokers [23]. In fact, any drug exposure during pregnancy, including alcohol, is associated with an increased risk for SUID [3]. Other risk factors include young maternal age $(<20$ years old).

\section{Protective Factors for SUID}

Breastfeeding

Breastfeeding has been shown to reduce the risk of various diseases including otitis media, necrotizing enterocolitis, asthma, diabetes, obesity, and SUID [24]. One meta-analysis demonstrated that breastfeeding for at least two months was protective for SUID, with a directly proportional relation with increased time of breastfeeding [25]. Partial and exclusive breastfeeding proved to be protective in infants who only slept in the supine position, although exclusive breastfeeding proved more protective than partial [20]. Because of all these described benefits, the term "breastsleeping” was coined. It tries to englobe the notion that maternal contact has a critical role in optimization of breastfeeding, and the fact that data and information should be gathered from studies of breastsleeping dyads, because of possible different outcomes from studies where no breast sleeping is performed [26].

Room Sharing

Room sharing is not a synonym of co-sleeping. It implies sleeping in the same room but on separate surfaces, albeit close to them. Studies have demonstrated that room sharing may reduce the risk of SUID in 


\section{Cureus}

half. Therefore, room sharing is essential for at least the first six months of life and recommended ideally during the first year [27].

Pacifier Use

The use of a pacifier before naptime and bedtime is accepted and recommended by the AAP, even though it may be related to cases of malocclusion and breastfeeding technique concerns. Multiple studies have yielded evidence that this intervention may reduce the risk of SUID from 50-90\% [27-28].

\section{Current recommendations}

In a 2016 update, the American Academy of Pediatrics published their new recommendations regarding safesleeping techniques to reduce the risk of SUID, emphasizing the need for supine positioning during sleep. The summary of these recommendations are outlined in the questionnaire below [3].

\section{Materials And Methods}

A cross-sectional study was performed in a second level, public, pediatric hospital in Mexico City, from May the 1st to July 30th of 2019. Subjects were selected via convenience sampling and data was collected by interviewing infant's parents or legal guardians. A series of specific questions were asked based on the AAP 2016 guidelines (Table 1), to depict whether the studied population followed the established recommendations or not. We focused on the most significant infant-related risk factors, since most of the maternal-related risk factors can't be controlled after the birth of the infant. Parents gave consent and agreed to participate in the direct verbal questionnaire. 


\section{Cureus}

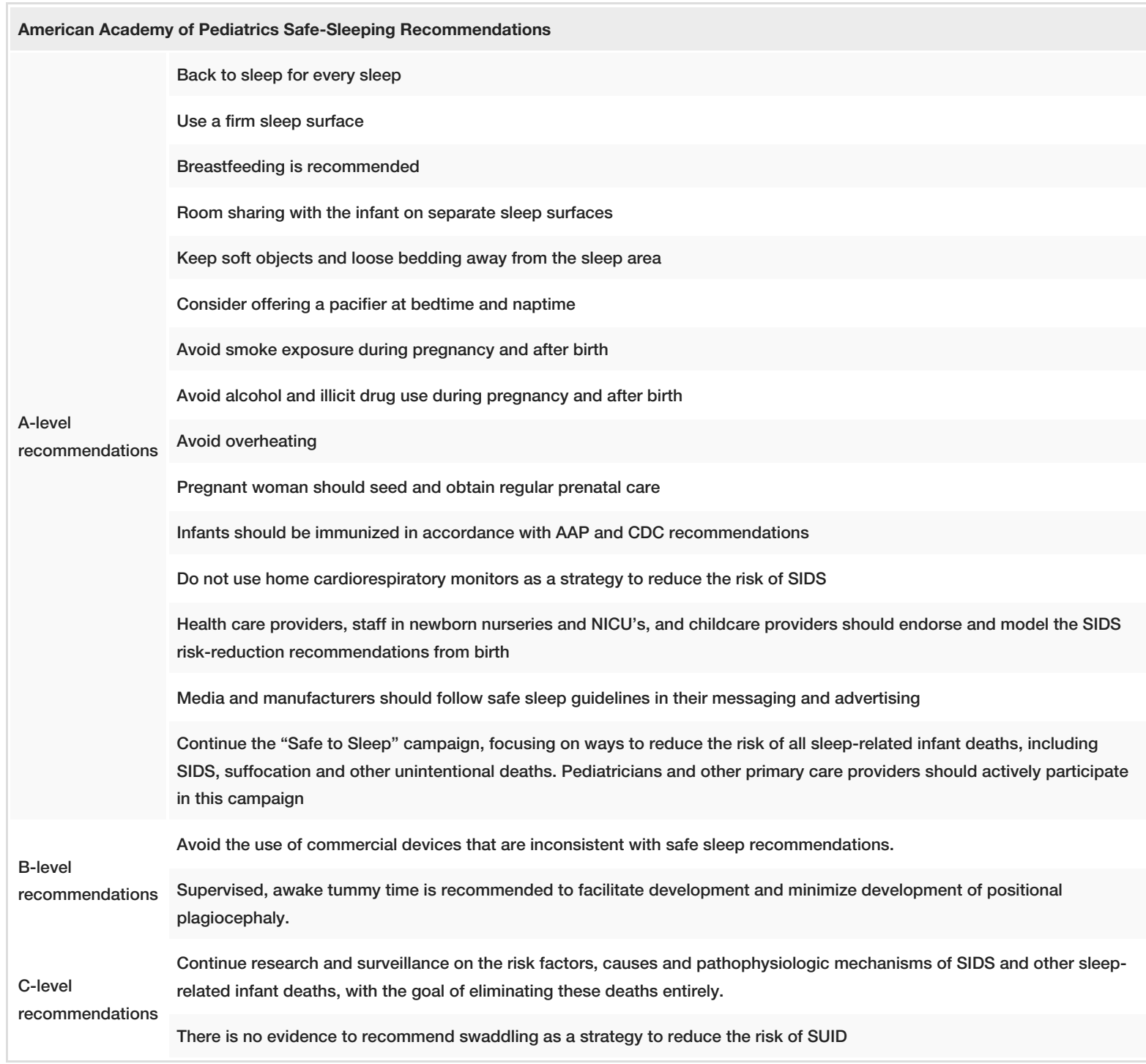

\section{TABLE 1: AAP 2016 guidelines with strength of recommendation to prevent SUID [3]}

AAP: American Association of Pediatrics.

SUID: Sudden Unexpected Infant Death

CDC: Centers for Disease Control

NICU: Neonatal Intensive Care Unit

Inclusion Criteria

To be included in the study, subjects had to be at least 1 day old, and less than 12 months old. We included all patients that presented in the out-patient clinic, in-patient hospitalization service, as well as those who presented to the emergency room.

Exclusion Criteria

Subjects older than 12 months were excluded from the study and those whose parents or legal guardians refused to answer the questionnaire. Patients with special care or nighttime monitoring were excluded as well.

Data was collected with a questionnaire encompassing infant-related risk factors for SUID, and it was answered by the parent or legal guardian of the infant. We asked the patient's legal guardian questions based 


\section{Cureus}

on the known risk factors for SUID (Table 2), such as co-sleeping, sleeping in a supine position, the use of pillows, sleeping environment characteristics, bedding and the use of a pacifier.

\begin{tabular}{|l|l|}
\hline SIDS Risk Factors & \\
\hline Maternal & Smoking during or after pregnancy \\
& Maternal age $<20$ years \\
& Inconsistent prenatal care \\
& Prone or side sleep position \\
& Loose bedding \\
Infant & Bed-sharing \\
& Low birth weight and prematurity \\
& Siblings with SUID
\end{tabular}

TABLE 2: Summary of SUID maternal and infant-related risk factors

SUID: Sudden Unexpected Infant Death

Statistical Analysis

The data collection was made in Microsoft Excel 2018 and further statistical analysis was performed in SPSS version 21.0 (IBM), and data for categorical variables is reported in frequencies and percentages, the numerical variables are expressed in means and standard deviations.

\section{Results}

A total of 184 infants were included in the analysis. The mean age of the study sample was 5.87 months (SD 3.18). There were 117 males, and 67 females. Of the 184 infants surveyed, the vast majority did not sleep alone, contrary to the AAP recommendations for safe sleeping (78.8\% did not sleep alone). Additionally, more than half of the infants did not sleep in a supine position, putting them at risk for SUID $(51.1 \%$ did not sleep supine) (Table 3).

$\begin{array}{lll}\text { Questionnaire Answers } & \text { Frequency }(\mathrm{n}=184) & \text { Percentage } \\ \text { Sleep Alone } & 39 & 21.20 \% \\ \text { Use Pillow } & 117 & 64 \% \\ \text { Stuffed Animals } & 18 & 9.80 \% \\ \text { Sleep Supine } & 90 & 48.90 \% \\ \text { Falls } & 24 & 13 \% \\ \text { Use Pacifier } & 5 & 2.70 \%\end{array}$

TABLE 3: . Results from the questionnaire looking for known risk factors for SUID and incidence of falls from bed.

SUID: Sudden Unexpected Infant Death

Overall, the amount of patients that follow safe sleeping practices in our sample was low, the mean number of positive answers in the questionnaire was 1.57 (SD 0.9), with no patients following all the recommendations in the questionnaire, and over $10 \%$ following no recommendations at all (Figure 1 ). 


\section{Cureus}



\section{FIGURE 1: Histogram showing the frequencies of each number of positive answers (amount of AAP recommendations followed) detected by the 6-question survey done in the present study.}

AAP: American Association of Pediatrics

\section{Discussion}

SUID remains an important public health problem, due to the high death burden. Many cases could be prevented if the corresponding risk factors had been avoided. Therefore, several strategies have been addressed, mainly by developed countries, to reduce the number of affected children. The data we found in our study is alarming, and the problem must be addressed by national health authorities.

Although there is uncertainty about the pathogenesis of this syndrome, the focus has shifted to prevention, especially of the modifiable risk factors, through specific campaigns, recommendations and guidelines by pediatric associations, based on extensive observation and reported studies. Regarding our work, the results obtained were not far from what we hypothesized in a low socioeconomic status population, which was the case of the sample obtained in this particular hospital. Other studies with similar populations had similar results regarding bed sharing and co-sleeping prevalence [29]. Like what other studies have found, our population could extrapolate to the findings in the African American, American Indian and Native Alaskan population, where similar sleeping customs are practiced [3]. However, there is no clear epidemiological data on this pathology in our country. There seems to be a correlation with better sleep practices in mothers with higher socioeconomic and educational background [30] which could be assessed in further studies with a different population.

It is important to note that after every interview with the parent or legal guardian, we explained the current recommendations and guidelines to the parents, the actions to avoid and the actions to implement. However, many of these current guidelines (such as avoiding co-sleeping with the infant, which is very prevalent in the Mexican population) are unpractical for our population, mainly due to social, economic and psychological situations, and changing this paradigm will be a challenge. Furthermore, safe sleeping campaigns must be conducted in our country to instruct this knowledge to current and future parents, preventing cases of SUID. Medical personnel will have to shift their focus to inform and make parents aware of these recommendations. Recommendations are relatively simple for most parents to follow, but the lack of information and knowledge on this topic, both by parents and health workers make it hard to understand their importance.

Up to this date, there is not a consensus or guideline regarding risk reduction strategies for SUID in our home country, and therefore, the population has never been informed of the possible interventions to 
reduce the risk, and believe that their current sleeping practices are safe and adequate.

\section{Conclusions}

It was demonstrated that our population did not know about the proper and safe sleeping techniques to avoid the known sleep-related risk factors associated with SUID. Unfortunately, this is still an understudied subject in our country and in the world. Mexico lacks concluding and reliable epidemiological information to measure the real prevalence and incidence of this public health problem. With this study we were able to demonstrate the impending need to implement a "Safe-Sleeping" campaign in our country, such as the ones carried out in other parts of the world, where there is conclusive evidence of the benefits of implementing such campaigns to prevent SUID in the future. Optimistically in a nearby future, health authorities in Mexico and other Latin-American countries will implement a strategy to make the AAP recommendations part of government health campaigns parallel to those of universal vaccination of children and exclusive breastfeeding for the first six months of life.

\section{Additional Information \\ Disclosures}

Human subjects: Consent was obtained by all participants in this study. Hospital Pediatrico Legaria issued approval NA. The Hospital in which we conducted the present study does not have a body that acts as an IRB due to the low funding and lack of research incentives in our health system. But the directors of the education office of the hospital was properly notified and approved the present study. Animal subjects: All authors have confirmed that this study did not involve animal subjects or tissue. Conflicts of interest: In compliance with the ICMJE uniform disclosure form, all authors declare the following: Payment/services info: All authors have declared that no financial support was received from any organization for the submitted work. Financial relationships: All authors have declared that they have no financial relationships at present or within the previous three years with any organizations that might have an interest in the submitted work. Other relationships: All authors have declared that there are no other relationships or activities that could appear to have influenced the submitted work.

\section{References}

1. Willinger M, James LS, Catz C: Defining the sudden infant death syndrome (SIDS): deliberations of an expert panel convened by the National Institute of Child Health and Human Development. Pediatr Pathol. 1991, 11:677-684. 10.3109/15513819109065465

2. Shapiro-Mendoza CK, Tomashek KM, Anderson RN, Wingo J: Recent national trends in sudden, unexpected infant deaths: more evidence supporting a change in classification or reporting. Am J Epidemiol. 2006, 163:762-769. 10.1093/aje/kwj117

3. Task force on sudden infant death syndrome: SIDS and other sleep-related infant deaths: updated 2016 recommendations for a safe infant sleeping environment. Pediatrics. 2016, 138:e20162938. 10.1542/peds.2016-2938

4. United States Department of Health and Human Services (US DHHS), Centers for Disease Control and Prevention (CDC), National Center for Health statistics. (2019). Accessed: July 2019: https://www.cdc.gov/nchs/index.htm.

5. Center for Disease Control and Prevention. Sudden unexpected infant death and sudden infant death syndrome. . (2019). Accessed: June 17: http://www.cdc.gov/sids/.

6. Willinger M, Hoffman HJ, Hartford RB: Infant sleep position and risk for sudden infant death syndrome: report of meeting held january 13 and 14, 1994, National Institutes of Health, Bethesda, MD. Pediatrics. 1994, 93:814-819.

7. Spinelli J, Collins-Praino L, Van Den Heuvel C, Byard RW: Evolution and significance of the triple risk model in sudden infant death syndrome. J Paediatr Child Health. 2017, 53:112-115. 10.1111/jpc.13429

8. Ostfeld BM, Schwartz-Soicher O, Reichman NE, et al.: Prematurity and sudden unexpected infant deaths in the United States. Pediatrics. 2017, 140:e20163334. 10.1542/peds.2016-3334

9. Malloy MH: Size for gestational age at birth: impact on risk for sudden infant death and other causes of death, USA 2002. Arch Dis Child Fetal Neonatal Ed. 2007, 92:F473-F478. 10.1136/adc.2006.107094

10. Mathews TJ, Menacker F, MacDorman MF: Infant mortality statistics from the 2001 period linked birth/infant death data set. Natl Vital Stat Rep. 2003, 52:1.

11. Guntheroth WG, Spiers PS: Sleeping prone and the risk of sudden infant death syndrome . JAMA. 1992, 267:2359-62.

12. Kassa H, Moon RY, Colvin JD: Risk factors for sleep-related infant deaths in in-home and out-of-home settings. Pediatrics. 2016, 138:e20161124. 10.1542/peds.2016-1124

13. Wong SB, Zhao LL, Chuang SH, et. al: Is prone sleeping dangerous for neonates? Polysomnographic characteristics and NDN gene analysis. Ci Ji Yi Xue Za Zhi. 2019, 31:113-117. 10.4103/tcmj.tcmj_29_18

14. Erck-Lambert AB, Parks SE, Cottengim C, et al.: Sleep-related infant suffocation deaths attributable to soft bedding, overlay, and wedging. Pediatrics. 2019, 143:e20183408. 10.1542/peds.2018-3408

15. Blair PS, Mitchell EA, Heckstall-Smith EMA, Fleming PJ: Head covering - a major modifiable risk factor for sudden infant death syndrome: a systematic review. Arch Dis Child. 2008, 93:778-83. 10.1136/adc.2007.136366

16. Salm Ward, TC, Doering JJ: Application of a socio-ecological model to mother-infant bed-sharing . Health Educ Behav. 2014, 41:577-589. 10.1177/1090198114543010

17. Marinelli, KA, Ball HL, McKenna JJ, Blair PS: An integrated analysis of maternal-infant sleep, breastfeeding, and sudden infant death syndrome research supporting a balanced discourse. J Hum Lact. 2019, 35:510-520. 
10.1177/0890334419851797

18. Baddock SA, Purnell MT, Blair PS, et. al. : The influence of bed-sharing on infant physiology, breastfeeding and behaviour: a systematic review. Sleep Med Rev. 2019, 43:106-117. 10.1016/j.smrv.2018.10.007

19. Carpenter R, McGarvey C, Mitchell EA, et al.: Bed sharing when parents do not smoke: is there a risk of SIDS? An individual level analysis of five major case-control studies. BMJ Open. 2013, 3:002299. 10.1136/bmjopen-2012-002299

20. Vennemann MM, Hense HW, Bajanowski T, et al.: Bed sharing and the risk of sudden infant death syndrome: can we resolve the debate?. J Pediatr. 2012; 160, 44-8. 10.1016/j.jpeds.2011.06.052

21. Fleming PI, Blair PS, Bacon C, et al.: Environment of infants during sleep and risk of the sudden infant death syndrome: results of 1993-5 case-control study for confidential inquiry into stillbirths and deaths in infancy. BMJ. 1996, 313:191-195. 10.1136/bmj.313.7051.191

22. Anderson TM, Lavista-Ferres JM, Ren SY, et al.: Maternal smoking before and during pregnancy and the risk of sudden unexpected infant death. Pediatrics. 2019, 143:e20183325. 10.1542/peds.2018-3325

23. Klonoff-Cohen HS, Edelstein SL, Lefkowitz ES, et al.: The effect of passive smoking and tobacco exposure through breast milk on sudden infant death syndrome. JAMA. 1995, 273:795-798. 10.1001/jama.1995.03520340051035

24. American Academy of Pediatrics: Breastfeeding and the use of human milk. Pediatrics. 2012, 129:e827-e841. 10.1542/peds.2011-3552

25. Thompson JMD, Tanabe K, Moon RY, et al.: Duration of breastfeeding and risk of SIDS: an individual participant data meta-analysis. Pediatrics. 2017, 140:e20171324. 10.1542/peds.2017-1324

26. McKenna JJ, Gettler LT: There is no such thing as infant sleep, there is no such thing as breastfeeding, there is only breastsleeping. Acta Paediatr. 2016, 105:17-21. 10.1111/apa.13161

27. Moon RY, Task Force on Sudden Infant Death Syndrome: SIDS and other sleep-related infant deaths: evidence base for 2016 updated recommendations for a safe infant sleeping environment. Pediatrics. 2016, 138:e20162940. 10.1542/peds.2016-2940

28. Mitchell EA, Blair PS, L'Hoir MP: Should pacifiers be recommended to prevent sudden infant death syndrome?. Pediatrics. 2006, 117:1755-1758. 10.1542/peds.2005-1625

29. Kendall-Tackett K, Cong Z, Hale TW: Mother-infant sleep locations and nighttime feeding behavior: U.S. data from the Survey of Mothers' Sleep and Fatigue. Clin Lactation. 2010, 1:27-31.

30. da Silva BGC, da Silveira MF, de Oliveira PD, et al.: Prevalence and associated factors of supine sleep position in 3-month-old infants: findings from the 2015 Pelotas (Brazil) Birth Cohort. BMC Pediatr. 2019, 19:165. 10.1186/s12887-019-1534-3 